\title{
Influência das variáveis climáticas na incidência da varíola do mamoeiro "Sunrise Golden" em diferentes densidades de cultivo sob manejo orgânico de produção
}

Thiago Cunha Silverio ${ }^{1}$

Luiz Aurélio Peres Martelleto

Nilson Costa Rezende ${ }^{3}$

Herton Chimelo Pivoto 4

Livia Israel Ferreira 5

\section{Resumo}

A doença fúngica conhecida como varíola ou pinta preta é um dos principais entraves para a produção comercial do mamão (Carica papaya) nas regiões tropicais e subtropicais. Sua elevada incidência em lavouras comerciais ou domésticas, além de promover a queda prematura de folhas, provoca danos na superfície dos frutos, afetando sua qualidade. A obtenção de informações sobre os efeitos das variáveis climáticas sobre as doenças é indispensável para recomendar o manejo racional. Assim, em um experimento com diferentes densidades de cultivo orgânico do mamoeiro instalado no estado do Espírito Santo, grande produtor de mamão do Brasil, foram registrados por meio de uma microestação meteorológica os valores diários das temperaturas e da umidade relativa do ar desde o transplantio. Os valores foram analisados nos meses de abril, maio, junho, julho e agosto de 2015, quando ocorreu a incidência natural da varíola nos mamoeiros. Para estabelecer as análises, adotaram-se notas de severidade da doença. As diferentes densidades de cultivo adotadas foram: (D1) 1.667, (D2) 2.167, (D3) 2.667, (D4) 3.167, (D5) 3.667 e (D6) 4.167 plantas por hectare. Os resultados apontaram que a maior severidade da doença ocorre devido a uma combinação de maiores amplitudes térmicas, média das temperaturas máximas variando de 28 a $30^{\circ} \mathrm{C}$, média das temperaturas mínimas próximas de $20^{\circ} \mathrm{C}$ e umidade relativa do ar próxima de 80 \%. 0 maior adensamento de cultivo também favorece a gravidade da doença.

Palavras-chave: Asperisporium caricae. Controle Alternativo. Carica papaya.

\section{Introdução}

O mamoeiro (Carica papaya) é uma frutífera de grande importância mundial. É cultivado principalmente nos países tropicais, possuindo destacável aceitação pelos consumidores (SANTOS, 2009). Para uma produção satisfatória e aumento das exportações brasileiras, a cultura do mamoeiro necessita superar uma série de problemas, especialmente os de natureza fitossanitária. Atualmente, o

1 Centro Estadual Integrado de Educação Rural (CEIER), professor mestre em Agricultura Orgânica. thiagocsilverio@gmail.com.

2 Universidade Federal Rural do Rio de Janeiro (UFRRJ), professor doutor em Fitotecnia. luizmarte@hotmail.com.

3 Centro Estadual Integrado de Educação Rural (CEIER), professor mestre em Agricultura Orgânica. nilsonrezende@yahoo.com.br.

4 Universidade Federal Rural do Rio de Janeiro (UFRRJ), mestre em Agricultura Orgânica. hpivoto@hotmail.com.

5 Centro Estadual Integrado de Educação Rural (CEIER), professora mestre em Metrologia. liviaf.ufrrj@yahoo.com.br. 
cultivo extensivo do mamoeiro, localizado e concentrado em algumas poucas regiões do Brasil, tem tornado a produção dependente do uso de agrotóxicos, especialmente fungicidas. Pelo fato de ainda não existirem variedades resistentes às doenças, em geral, a dependência com relação ao controle químico é ainda maior (SANTOS, 2013).

Com relação à distribuição geográfica das produções mais destacáveis no Brasil, observa-se uma grande faixa contínua, próxima ao litoral, que vai de Linhares (Espírito Santo) até Porto Seguro (Bahia). Todavia, conforme destacam Almeida et al. (2013), nos últimos anos, nota-se uma progressiva migração da cultura do mamoeiro para o interior do país, devido, principalmente, às questões ligadas a problemas de ordem fitossanitária.

A varíola (Asperisporium caricae) se destaca como um dos principais entraves para a produção de mamão no noroeste do Espírito Santo. Sua elevada incidência em lavouras comerciais ou domésticas constitui-se em um dos principais problemas fitossanitários da cultura nesse estado (SILVERIO et al., 2015).

A primeira descrição da varíola no Brasil tem mais de 100 anos e foi realizada por Maublanc (1913), citado por Martelleto (2007), no Estado do Rio de Janeiro. Atualmente, no Brasil, é uma doença comum nas regiões produtoras de maior importância do país (Espírito Santo, Bahia, Minas Gerais, Ceará e Paraná) (MARTELLETO, 2009). A varíola [Asperisporium caricae (Speg.) Maubl.] incita manchas em folhas e frutos levando a lesões necróticas. 0 nível de incidência varia em decorrência de diversos fatores, com destaque para a temperatura e a umidade.

Apesar da importância das doenças fúngicas para o mamoeiro, como o tombamento (ou damping-off), Podridão-de-Phytophthora, Antracnose, oídio, mancha chocolate, podridão preta, mancha de Corynespora e varíola, os estudos são incipientes, porém com informações seguras sobre o efeito dos fatores climáticos que atuam durante o progresso de epidemias dessas doenças. No presente trabalho objetivou-se avaliar o efeito das variáveis climáticas relacionadas com diferentes densidades de cultivo do mamoeiro.

\section{Material e métodos}

O estudo foi conduzido no Centro Estadual Integrado de Educação Rural (CEIER), situado no município de Águia Branca, Região Noroeste do Estado do Espírito Santo (altitude de 130 m, latitude 18059'01" S e longitude W. Gr, 40 44' 22" W) (Programa de Assistência Técnica e Extensão Rural - PROATER, 2011).

O clima é tropical com estação seca (classificação climática de Köppen-Geiger: Aw). A temperatura média anual situa-se entre 30 e $34^{\circ} \mathrm{C}$. As precipitações anuais atingem, em média, mais de $3,5 \mathrm{~mm} / \mathrm{dia}^{-1}$, porém a maior causa de preocupação é a distribuição irregular das chuvas na região (PROATER, 2011).

Adotou-se o delineamento experimental de blocos ao acaso, com seis densidades de cultivo diferentes e quatro repetições ou blocos. As diferentes densidades de cultivo adotadas foram: (D1)

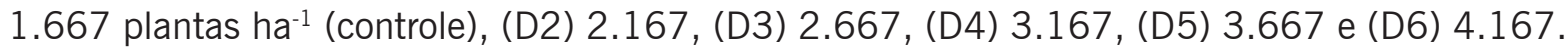

As parcelas com o espaçamento $3 \times 2$ m ocuparam uma área de $36 \mathrm{~m}^{2}$ (densidade de 1.667 plantas ha-1); as parcelas com espaçamento $3 \times 1,54 \mathrm{~m}$ ocuparam uma área de 27,72 $\mathrm{m}^{2}$ (densidade de 2.167 plantas ha-1); as parcelas com espaçamento $3 \times 1,25 \mathrm{~m}$ ocuparam uma área de 22,5 $\mathrm{m}^{2}$

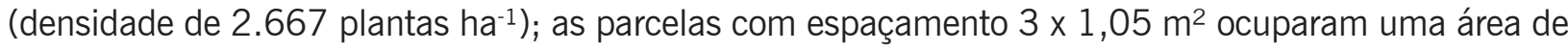
18,9 $\mathrm{m}^{2}$ (densidade de 3.167 plantas ha-1); as parcelas com espaçamento $3 \times 0,9 \mathrm{~m}$ ocuparam uma área de 16,2 $\mathrm{m}^{2}$ (densidade de 3.667 plantas ha-1 $^{-1}$; e as parcelas com espaçamento de $3 \times 0,8 \mathrm{~m}$ 
ocuparam uma área de 14,4 $\mathrm{m}^{2}$ (densidade de 4.167 plantas ha-1 ${ }^{-1}$. A variedade do mamoeiro utilizada no experimento foi a "Sunrise Golden".

Instalou-se no local do experimento uma microestação meteorológica, por meio da qual obteve-se o registro em todo o período de análise, das variações diárias de temperatura do ar. A estação foi fixada em um suporte de madeira, posicionada para o sul, de forma a evitar exposição direta dos raios solares.

0 instrumento foi instalado no centro do local de cultivo, a cerca de 1,80 m do nível do solo. Os dados de umidade relativa do ar foram registrados pela mesma estação meteorológica e coletados por volta das $8 \mathrm{~h}$ de dias sem chuva e registrou-se a média dos valores dentro de cada mês. Foram realizados os confrontos entre as variáveis climáticas: média das temperaturas máximas, temperaturas mínimas, amplitudes térmicas e umidade relativa do ar, medidas diariamente no local do experimento.

Os valores foram analisados nos meses de abril, maio, junho, julho e agosto de 2015, quando ocorreu a incidência natural da varíola nos mamoeiros. Para estabelecer as análises de regressão, nos meses citados, adotaram-se as notas de severidade da doença, pelos danos causados na sexta, na décima e na décima quarta folha, a contar da mais recém-emergida, denominada de "folha-índice".

Os registros de ocorrência da doença seguiram a padronização (por meio de comparação) das lesões necróticas, com a escala diagramática de notas em função da severidade da varíola, conforme Barreto et al. (2011) e adaptada para nível de severidade para a experimentação.

A escala diagramática de notas expressando os níveis de severidade no folíolo lesionado foi estabelecida da seguinte maneira: $0=$ folha sem lesões; $1=$ até $0,25 \% ; 2=1,75 \% ; 3=7,5 \%$; $4=19 \% ; 5=30 \% ; 6=$ com mais de $31 \%$.

Posteriormente, nas análises estatísticas, as notas médias atribuídas foram transformadas novamente em porcentual de área foliar afetada, para um melhor aferimento da severidade da varíola. Adotou-se a folha-índice 10, citada como referência, por ser aquela que normalmente tem a flor principal em antese em sua axila. A folha 10 foi a que possibilitou tirar as melhores inferências e, também, ser facilmente localizada na planta. A partir dessas análises, estabeleceram-se as equações de regressão.

A análise estatística foi conduzida por meio de análise da variância e teste de hipóteses e adotou-se a média dos 12 (doze) meses de avaliação (setembro/2014 a agosto/2015) para verificar a significância dos efeitos principais e da interação. Os resultados obtidos foram submetidos à análise de regressão polinomial.

\section{Resultados e discussão}

Os valores de severidade da varíola na folha-índice 10 possibilitaram tirar as melhores inferências e foram, portanto, adotados para estabelecer as correlações com as variáveis climáticas. A folha-índice 10 normalmente tem a flor principal em antese em sua axila. Os registros dos dados climáticos de temperatura máxima, temperatura mínima, amplitude térmica e umidade relativa durante a vigência do experimento foram expostos na Tabela 1 e os resultados na Figura 1. 
Tabela 1. Dados de temperatura: máximas, mínimas, médias e amplitude térmica; umidade relativa do ar registrada na área experimental durante o primeiro ano de cultivo dos mamoeiros, Águia Branca/ES (2014/2015)

\begin{tabular}{|c|c|c|c|c|c|}
\hline \multirow{2}{*}{ Meses/Ano } & \multicolumn{4}{|c|}{ Temperaturas $\left({ }^{\circ} \mathrm{C}\right)$} & \multirow{2}{*}{ UR (\%) } \\
\hline & Máximas & Mínimas & Médias & Amplitudes Térmicas & \\
\hline Set/2014 & 30,7 & 17,4 & 24,1 & 13,3 & 74 \\
\hline Out/2014 & 31,0 & 18,6 & 24,8 & 12,4 & 76 \\
\hline Nov/2014 & 31,5 & 20,4 & 26,0 & 11,1 & 78 \\
\hline Dez/2014 & 33,4 & 20,9 & 27.2 & 12,5 & 77 \\
\hline Jan/2015 & 36,6 & 21,3 & 29,0 & 15,3 & 69 \\
\hline Fev/2015 & 35,4 & 21,5 & 28,5 & 13,9 & 76 \\
\hline Mar/2015 & 34,1 & 21,4 & 27,8 & 12,7 & 78 \\
\hline Abr/2015 & 32,5 & 20,0 & 26,3 & 12,5 & 79 \\
\hline Mai/2015 & 29.5 & 17,9 & 23,7 & 11,6 & 80 \\
\hline Jun/2015 & 22,5 & 17,5 & 20,0 & 5,0 & 81 \\
\hline $\mathrm{Jul} / 2015$ & 21,5 & 17,0 & 19,3 & 4.5 & 82 \\
\hline Ago/2015 & 21,2 & 15,5 & 18,4 & 5,7 & 77 \\
\hline Médias & 30.0 & 19.1 & 24.6 & 10.9 & 77 \\
\hline
\end{tabular}

Fonte: Thiago Cunha Silverio (2014/2015).

Figura 1. Equações de regresșão entre variáveis climáticas e severidade da varíola na folha-índice 10 dos mamoeiros “Sunrise Golden”, Águia Branca/ES, 2015.
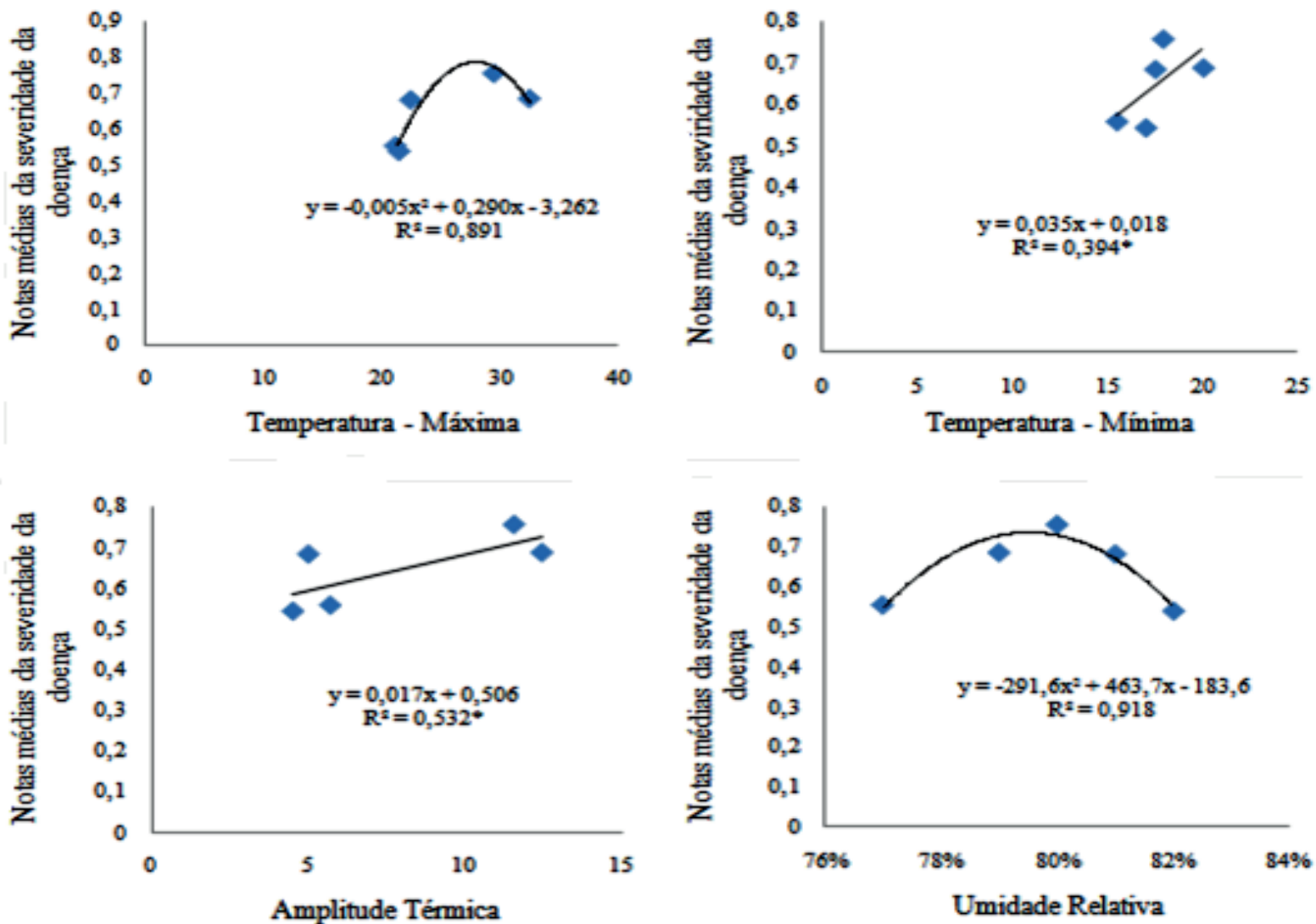

Fonte: Thiago Cunha Silverio (2015). 
Segundo as notas adotadas na folha-índice 10, os níveis de severidade da varíola do mamoeiro ao longo do primeiro ano de cultivo sofreram forte influência dos fatores climáticos. Assim, observa-se que a temperatura máxima e a umidade relativa do ar versus a severidade da doença se ajustam ao modelo matemático de regressão polinomial (quadrática) (FIGURA 1).

Com os modelos de regressão quadráticas pode-se inferir o pico da severidade da varíola do mamoeiro, por meio do monitoramento da temperatura máxima e da umidade relativa do ar.

Portanto, a combinação das condições média das temperaturas máximas próximas de $29,5^{\circ} \mathrm{C}$ e umidade relativa do ar próxima de 80 \% favorecem a epidemia da varíola.

Esses resultados concordam com os de Suzuki et al. (2007), que concluíram que para a pinta-preta as condições favoráveis ao desenvolvimento de epidemias correspondem à temperatura variando de 25 a $30^{\circ} \mathrm{C}$ e umidade relativa variando de 80 a $100 \%$.

Com relação à amplitude térmica, os resultados sugerem que, dentro das faixas anotadas, as maiores diferenças entre as temperaturas registradas durante o dia contra as registradas durante a noite favorecerão a ocorrência da varíola.

Sabe-se que as situações mais vigentes para localidades de maiores altitudes até certos limites possibilitam maiores amplitudes térmicas. Esta condição combinada com as outras variáveis destacadas são extremamente favoráveis à doença, justificando os níveis altos da varíola na região onde fica o município de Águia Branca, embora esteja a cerca de 150 metros acima do nível do mar.

No que se refere às diferentes densidades de cultivo, 1.667 (padrão comercial) e 2.167 plantas ha $^{-1}$ caracterizaram-se por valores próximos a zero em agosto de 2015 , indicando a significativa influência dos fatores climáticos sobre a epidemia da doença. Como pode ser verificado com os dados de temperatura e UR (\%) coletados no período (TABELA 1), cuja máxima foi de $21^{\circ} \mathrm{C}$ e mínima de 15 ${ }^{\circ} \mathrm{C}$ e a UR (\%) de $77 \%$, que são condições desfavoráveis a proliferação e desenvolvimento do fungo Asperisporium caricae.

De acordo com os dados meteorológicos coletados no período da experimentação, a severidade da varíola é favorecida, principalmente, nos meses de março, abril e maio de 2015, demonstrado na Figura 2.

Figura 2. Severidade da varíola, medida na folha-índice 10, para as diferentes densidades de cultivo do mamoeiro ao longo de um de monitoramento, Águia Branca/ES, 2015

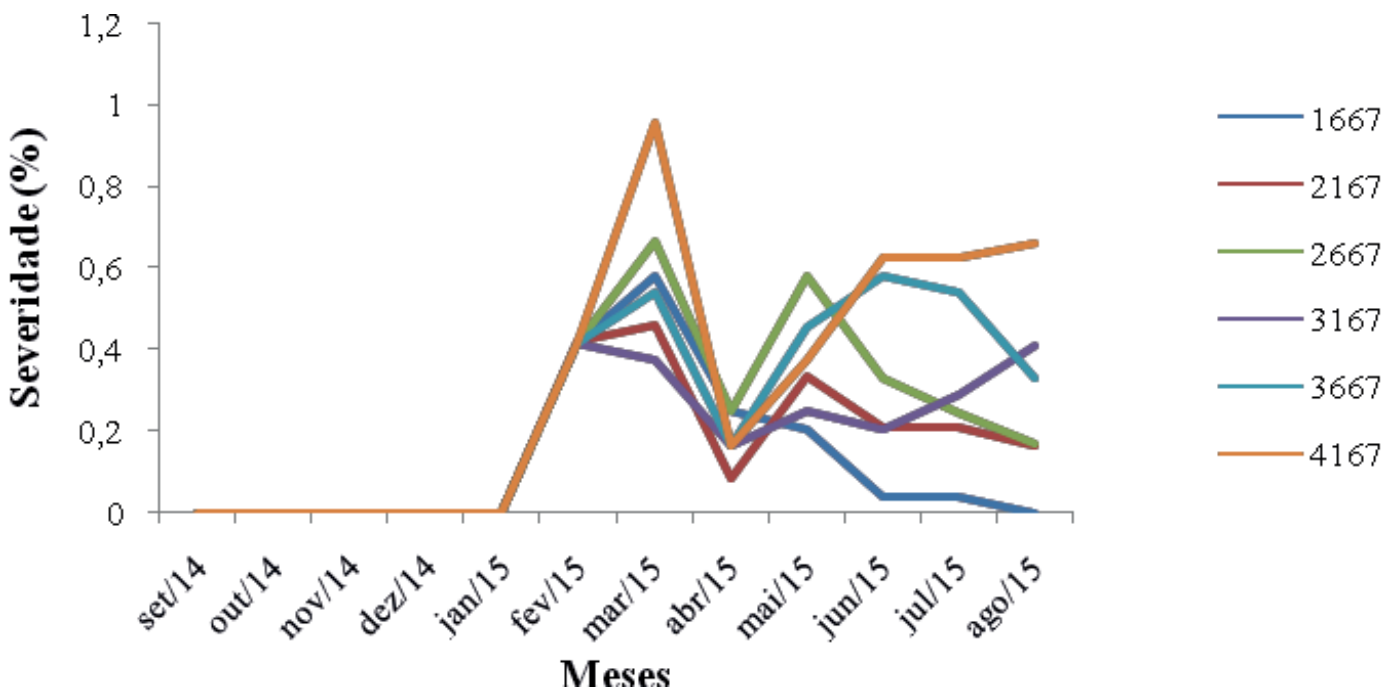

Fonte: Thiago Cunha Silverio (2015). 
A manifestação da doença com maior severidade nos meses mais quentes e chuvosos do ano ratifica o que Martelleto (2009) encontrou em sua pesquisa, em que nos meses mais frios a incidência da doença nas folhas do mamoeiro é ínfima e nos meses mais quentes (janeiro, fevereiro e março) há um aumento abrupto na severidade da doença.

Os resultados descritos corroboram os estudos de Vivas (2009) e Suzuki et al. (2007), que descreveram que a varíola é mais severa nos meses mais quentes do ano na Região Sudeste do Brasil.

Observa-se na Tabela 2 a correlação da severidade da varíola nas folhas versus a severidade nos frutos do mamoeiro.

Tabela 2. Coeficientes de correlação de Pearson ${ }^{1}(r)$ entre a severidade da varíola nas folhas versus a severidade nos frutos, Águia Branca/ES, 2014/2015.

\begin{tabular}{|c|c|c|c|c|c|c|c|c|c|}
\hline & \multicolumn{3}{|c|}{$\%$ de leite na calda } & \multicolumn{6}{|c|}{ Densidades de cultivo } \\
\hline & $0 \%$ & $5 \%$ & $10 \%$ & 1667 & 2167 & 2667 & 3167 & 3667 & 4167 \\
\hline Correlações & $0,82^{* *}$ & $0,76^{* *}$ & $0,84^{* *}$ & $0,83^{* *}$ & $0,78^{* *}$ & $0,72^{* *}$ & $0,74^{* *}$ & $0,75^{* *}$ & $0,74^{* *}$ \\
\hline
\end{tabular}

Correlações baseadas no confronto de subparcelas entre a quantidade de doença nas folhas com os frutos (valores relativos a 24 pareamentos para \% de leite na calda e 12 pareamentos para densidades de cultivo); **Significativo a $0,2 \%$ de probabilidade $(P<0,002)$.

Fonte: Thiago Cunha Silverio (2015).

Observa-se que existe influência da infecção do fungo no fruto se ocorrer maior fonte de inóculo nas folhas. Esses resultados são semelhantes aos que podem ser computados a partir dos dados de Dianese et al. (2007) e Suzuki et al. (2007), que estudaram, respectivamente, a reação de genótipos de mamoeiro à varíola em Brasília e a severidade nas folhas e nos frutos em diferentes formas de controle dessa moléstia. Sendo assim, se as folhas do mamoeiro estiverem acometidas pela infecção do fungo Asperisporium caricae, os frutos também serão acometidos, e a severidade se dará pelo grau de infecção do fungo nas folhas.

\section{Conclusões}

Nas densidades de cultivo com uma elevada população de plantas há uma grande variação na incidência da doença, uma vez que no adensamento populacional há uma maior concentração de umidade, sendo assim, há um favorecimento à infecção secundária da doença no pomar.

A padronização, por meio de escala diagramática, do número de lesões necróticas nas folhas-índice demonstra que ocorreu o estabelecimento de um microclima favorável para o fungo, Asperisporium caricae, nas densidades de cultivo mais adensadas.

A aferição da UR (\%) e da temperatura é uma importante alternativa para os produtores entrarem com as medidas de controle em suas lavouras, uma vez que os balizadores se concentram em altas temperaturas com altas taxas de umidade relativa do ar. 


\title{
Influence of climatic variables on the incidence of smallpox of "sunrise golden" papaya in different densities of cultivation under organic production management
}

\begin{abstract}
The fungal disease known as smallpox or black spot is one of the main obstacles for the commercial production of papaya (Carica papaya) in tropical and subtropical regions. Its high incidence in commercial or domestic crops, besides promoting the premature fall of leaves, causes damages on the surface of the fruits, affecting their quality. Obtaining information on the effects of climatic variables on diseases is indispensable to recommend their rational management. Therefore, in an experiment with different densities of organic cultivation of papaya assembled in the state of Espírito Santo, a major producer of papaya in Brazil, the daily values of Temperatures and Relative Humidity of the Air were recorded through a meteorological microestation from the transplanting. The values were compared for the months of April, May, June, July and August of 2015, when the natural incidence of smallpox occurred in the mammals. To establish the analyzes, the severity of the disease was evaluated. The different cultivation densities adopted were: (D1) 1.667, (D2) 2.167, (D3) 2.667, (D4) 3.167, (D5) 3.667 and (D6) 4.167 plants per hectare. The results showed the highest severity of the disease occurs in a combination of higher Thermal Amplitudes, average Maximum Temperatures ranging from 28 to $30{ }^{\circ} \mathrm{C}$, Average Minimum Temperatures close to $20{ }^{\circ} \mathrm{C}$ and Relative Air Humidity close to $80 \%$. The greater density of cultivation is favored by the disease seriousness.
\end{abstract}

Keywords: Asperisporium caricae. Alternative Control. Carica papaya.

\section{Referências}

ALMEIDA, G. V. B.; RODRIGUES, M. G. V.; CASTRICINI, A. Produção, mercado e aspectos econômicos. Informe Agropecuário; Belo Horizonte, MG, v. 34, n. 275, 2013.

BARRETO, L. F.; SAVAN, P. A. L.; LIMA, L. L.; LODO, B. N. Avaliação de fungicidas no controle de Asperisporium caricae na cultura do mamoeiro. Revista Brasileira de Fruticultura, Jaboticabal - SP, v. 33, n. Esp. p. 399-403, out./2011. Disponível em: < http://www.scielo.br/ pdf/rbf/v33nspe1/a51v33nspe1.pdf>. Acesso em: 07 jan. 2019.

DIANESE, A. C.; BLUM, L. E. B.; DUTRA, J. B.; LOPES, L. F.; SENA, M. C.; FREITAS, L. F.; YAMA$\mathrm{NISHI}, \mathrm{O}$. K. Reação de genótipos de mamoeiro à varíola e à podridão-do-pé. Brasília, 2007. Disponível em: <http://www.scielo.br/pdf/fb/v32n5/v32n5a08.pdf>. Acesso em: 05 out. 2017.

INSTITUTO CAPIXABA DE PESQUISA ASSISTÊNCIA TÉCNICA E EXTENSÃO RURAL (INCAPER). PROATER - Programa de Assistência Técnica e Extensão Rural do Incaper de Águia Branca - INCAPER, Águia Branca - ES, 2011. Disponível em: < https://incaper.es.gov.br/media/incaper/proater/ municipios/Noroeste/Aguia Branca.pdf>. Acesso em: 07 jan. 2019. 
MARTElleto, L. A. P.; RIBEIRO, R. L. D.; Carmo, M. G. F. do; SudomARTElleto, M.; GOES, A. Incidência da varíola, causada por Asperisporium caricae, em folhas de mamoeiros submetidos ao manejo orgânico, em diferentes ambientes de cultivo. Summa Phytopathologica (Impresso), v. 35, p. 288-292, 2009.

MAUBLANC, A. Uma moléstia do mamoeiro (Carica papaya L.). Boletim do Ministério da Agricultura, Indústria e Comércio, Brasília, v. 2, n. 1, p. 126-130, 1913.

SANTOS, P. H. D. Produtos alternativos no controle de doenças fúngicas em folha e fruto de mamoeiro. 2013. Dissertação (Mestrado - Produção Vegetal) - Universidade Estadual do Norte Fluminense Darcy Ribeiro, Centro de Ciências e Tecnologias Agropecuárias, Campos dos Goytacazes, RJ, 2013.

SANTOS, V. J. Avaliação de resistência de genótipos de mamoeiro Asperisporium caricae. 2009. Dissertação (Mestrado), Universidade Federal do Recôncavo da Bahia- Cruz das Almas, BA, Centro de Ciências Agrárias, Ambientais e Biológicas, Universidade Federal do Recôncavo da Bahia, 2009.

SilveriO, T. C.; ReZENDE, N. C.; PIVOTO, H. C.; SAlLeS, R. E.; MARTELLETO, L. A. P. Ação do leite de vaca adicionado à calda bordalesa, associado a diferentes densidades de cultivo, na ocorrência da varíola do mamoeiro. Cadernos de Agroecologia, v. 10, n. 3, maio 2015. ISSN 2236-7934. Disponível em: <http://aba-agroecologia.org.br/revistas/index.php/cad/article/view/17859>. Acesso em: 05 out. 2017.

SUZUKI, M. S.; ZAMBOLIM, L.; LIBERATO, J. R. Progresso de doenças fúngicas e correlação com variáveis climáticas em mamoeiro. Summa Phytopathologica, v. 33, n. 2, p. 167-177, 2007. Disponível em: <http://www.scielo.br/pdf/sp/v33n2/a11v33n2.pdf>. Acesso em: 07 jan. 2019.

VIVAS, M. Avaliação de germoplasma e híbridos de mamoeiro quanto à resistência às doenças causadas pelos fungos Asperisporium caricae, Colletotrichum gloeosporioides, oidium caricae e Phoma caricae-papayae. 2009. 103 f. Dissertação (Mestrado em Genética e Melhoramento de Plantas) Universidade Estadual do Norte Fluminense - UENF, 2009.

Submetido em: 18/04/2017.

Aceito em: 16/10/2017. 
Como citar:

ABNT

SilVerio, T. C.; MARTELleto, L. A. P.; ReZEndE, N. C.; PIVOtO, H. C.; FERREIRA, L. I. Influência das variáveis climáticas na incidência da varíola do mamoeiro "Sunrise Golden" em diferentes densidades de cultivo sob manejo orgânico de produção. Revista Agrogeoambiental, Pouso Alegre, v. 10, n. 2, p. 75-84, dez. Doi: http://dx.doi.org/10.18406/2316-1817v10n420181208

APA

Silverio, T.C., martelleto, L. A. P., Rezende, N. C., PIVOto, H. C. \& FerreiRA, L. I. (2018). Influência das variáveis climáticas na incidência da varíola do mamoeiro "Sunrise Golden" em diferentes densidades de cultivo sob manejo orgânico de produção. Revista Agrogeoambiental, 10 (2), 75-84. Doi: http://dx.doi.org/10.18406/2316-1817v10n420181208

ISO

SILVERIO, T. C.; MARTELleto, L. A. P.; REZENDE, N. C.; PIVOTO, H. C. E FERREIRA, L. I. Influência das variáveis climáticas na incidência da varíola do mamoeiro "Sunrise Golden" em diferentes densidades de cultivo sob manejo orgânico de produção. Revista Agrogeoambiental, 2018, vol. 10, n. 2, pp. 75-84. Eissn 2316-1817. Doi: http://dx.doi.org/10.18406/2316-1817v10n420181208

\section{VANCOUVER}

Silverio TC, Martelleto LAP, Rezende NC, Pivoto HC, Ferreira LI. Influência das variáveis climáticas na incidência da varíola do mamoeiro "Sunrise Golden" em diferentes densidades de cultivo sob manejo orgânico de produção. Rev agrogeoambiental. 2018 abr.jun.; 10(2): 75-84. Doi: http://dx.doi.org/10.18406/2316$1817 v 10 n 420181208$ 